\title{
Workshop - Open Source Software for Computer Games and Virtual Worlds: Practice and Future
}

\author{
Per Backlund ${ }^{1}$, Björn Lundell ${ }^{1}$, and Walt Scacchi ${ }^{2}$ \\ ${ }^{1}$ University of Skövde, Sweden \\ ${ }^{2}$ University of California, Irvine, USA \\ \{per.backlund, bjorn. lundell\}@his.se, wscacchi@ics.uci.edu \\ http://www.his.se, http://cgvw.ics.uci.edu/
}

\section{Introduction}

Computer games and virtual worlds are increasingly used throughout our society with people playing on the bus, at home and at work. Computer games thus affect larger and larger number of people and areas in the society of today. There are even scholars who advocate that games or virtual environments create better environments for learning than traditional classrooms. This situation motivates the use of games and game technology for additional purposes, e.g. education, training, health care or marketing.

This new use distinguishes between entertainment games and games for other uses, with the term Serious Games being the most common for the latter category. Although the term itself is well established in both academia and industry, there is no current single definition of the concept. However, a common component of these definitions is that is the addition of pedagogy (activities that educate or instruct, thereby imparting knowledge or skill) that makes games serious. For the purpose of this workshop we define Serious Games as:

Serious Games are games that engage users in their pursuit, and contribute to the achievement of a defined purpose other than pure entertainment (whether or not the user is consciously aware of it).

A Serious Game can be achieved through a spectrum ranging from the mere utilisation of game technology for non-entertainment purposes to development of specifically designed games for some non-entertainment purpose or the use and/or adaptation of commercial games for non-entertainment purposes. We also propose that any combination of the above would constitute a feasible way to achieve the desired effect.

There are numerous examples of serious games and virtual worlds from various sectors. But what are the possible roles that Open Source Software can take in facilitating the development of a new generation of these technologies or applications? Many new games and virtual worlds are being created through the use of open source game engines and game asset creation tools. Game modding, itself an idea that often relies on retail computer games that are packaged with software development kits to create new game variants, are generally licensed for non-commercial redistribution with game mod source code using a open source software license. But the intersection of Open Source, Games and Virtual Worlds is perhaps just beginning. More ideas are 
being pursued, including how to facilitate games that rely on user-created content, or that incorporate social media (e.g., YouTube videos, Flickr photos, audio recording remixes), and social networking services to create new modes of game play. Games, Virtual Worlds, and Open Source Software also help serve the needs of independent game developers and virtual world developers who work with limited resources, outside large commercial enterprises.

\section{Workshop Aim}

The workshop aims to bring people from the Open Source and Serious Games communities together to discuss the current status of the area and to find a common future where the two areas can enrich each other.

Open Source Software for Computer Games and Virtual Worlds: Practice and Futures will feature position statements and presentations which will be open for discussion.

Suggested topics include, but are not limited to the following:

- Current examples of game or virtual world applications for learning, health, energy, environment, manufacturing or other areas

- Current examples of open source tools and techniques for creating games or virtual world applications

- Practical and theoretical perspectives of open source in games or virtual worlds

- Open source approaches to game modding and user created content

- The intersection of game culture and free/open source software culture

- Innovative combinations of game play mechanics, social media, and social networking, and open source software

- Experience in developing games or virtual worlds using open source software tools, techniques, concepts, or game engine, such as OGRE, OpenSim, Open Croquet, Irrlicht, Doom/Quake, etc.

\section{Program Committee}

- Per Backlund, University of Skövde, Sweden (workshop co-organiser)

- Bjorn Lundell, University of Skövde, Sweden (workshop co-organiser)

- Walt Scacchi, University of California Irvine, USA (workshop co-organiser)

- Rosario de Chiara, University of Salerno, Italy

- Henrik Gustavsson, University of Skövde, Sweden

- Vittorio Scarano, University of Salerno, Italy

- Robert J. Stone University of Birmingham and HFI DTC, UK 